\title{
Evaluation of microRNA expression in a sheep model for lung fibrosis
}

\author{
Udari Eshani Perera ${ }^{*}$, Habtamu B. Derseh ${ }^{1}$, Sasika N. V. Dewage ${ }^{2}$, Andrew Stent ${ }^{3}$, Rukmali Wijayarathna ${ }^{4}$ and \\ Kenneth J. Snibson ${ }^{1 *}$
}

\begin{abstract}
Background: Idiopathic pulmonary fibrosis (IPF) is a chronic progressive fibroproliferative disorder that has one of the poorest prognoses amongst interstitial lung diseases. Recently, the finding of aberrant expression levels of miRNAs in IPF patients has drawn significant attention to the involvement of these molecules in the pathogenesis of this disease. Clarification of the differential expression of miRNAs in health and disease may identify novel therapeutic strategies that can be employed in the future to combat IPF. This study evaluates the miRNA expression profiles in a sheep model for lung fibrosis and compares them to the miRNA profiles of both IPF patients and the mouse bleomycin model for pulmonary fibrosis. Pathway enrichment analyses were performed on differentially expressed miRNAs to illustrate which biological mechanisms were associated with lung fibrosis.

Results: We discovered 49 differentially expressed miRNAs in the sheep fibrosis model, in which 32 miRNAs were significantly down regulated, while 17 miRNAs were significantly upregulated due to bleomycin-induced lung injury. Moreover, the miRNA families miR-29, miR-26, miR-30, let-7, miR-21, miR-19, miR-17 and miR-199 were aberrantly expressed in both sheep and mouse models, with similar differential miRNAs expression observed in IPF cases. Importantly, 18 miRNAs were aberrantly expressed in both the sheep model and IPF patients, but not in mice.
\end{abstract}

Conclusion: Together with pathway enrichment analyses, these results show that the sheep model can potentially be used to characterize previously unrecognized biological pathways associated with lung fibrosis.

Keywords: Sheep model, microRNA, Idiopathic pulmonary fibrosis, Bleomycin

\section{Background}

Idiopathic pulmonary fibrosis (IPF) is a chronic progressive fibroproliferative disorder categorized under the umbrella of interstitial lung diseases [1-3]. Despite significant progress in understanding the pathogenic mechanisms of the disease, the molecular pathogenesis of the disease remains unclear [3]. MicroRNAs (miRNAs) are known to play critical roles in modifying a wide range of biological processes, including tissue development and differentiation; cellular proliferation; tissue repair; and

\footnotetext{
*Correspondence: udari.perera@unimelb.edu.au; ksnibson@unimelb.edu.au ${ }^{1}$ School of Veterinary Science, The University of Melbourne, Parkville, VIC, Australia

Full list of author information is available at the end of the article
}

regulation of intracellular cell signaling pathways. Many of these processes are frequent changes that occur in a variety of diseases, including $\operatorname{IPF}[2,4,5]$.

miRNAs are phylogenetically conserved small noncoding short RNA sequences, 19-22 nucleotides in length, that are generated by a Dicer enzyme from hairpin-shaped single-stranded RNA precursors composed of about 70-90 base pairs [2, 3, 6, 7]. Each miRNA has several targets and several microRNA can also regulate the same gene [2]. The main function of microRNA is to inhibit protein synthesis either by inhibiting the translation, or degradation, of mRNA $[1,5,8]$.

Recently, miRNA has drawn significant attention due to its aberrant expression in human IPF. Studies

(C) The Author(s). 2021 Open Access This article is licensed under a Creative Commons Attribution 4.0 International License, which permits use, sharing, adaptation, distribution and reproduction in any medium or format, as long as you give appropriate credit to the original author(s) and the source, provide a link to the Creative Commons licence, and indicate if changes were made. The images or other third party material in this article are included in the article's Creative Commons licence, unless indicated otherwise in a credit line to the material. If material is not included in the article's Creative Commons licence and your intended use is not permitted by statutory regulation or exceeds the permitted use, you will need to obtain permission directly from the copyright holder. To view a copy of this licence, visit http://creativecommons.org/licenses/by/4.0/ The Creative Commons Public Domain Dedication waiver (http://creativecommons.org/publicdomain/zero/1.0/) applies to the data made available in this article, unless otherwise stated in a credit line to the data. 
published over the last 10 years report that approximately $10 \%$ of miRNAs are altered in an IPF setting [4, 9]. However, the investigation of miRNAs in IPF is still at an early stage [3]. Even though microRNA expression profiles have been reported, miRNA-mRNA interactions and pathway enrichment related to IPF have yet to be comprehensively explored [3]. Increased understanding of miRNA expression profiles and their influence on biological process in IPF is therefore important for identification of potential biomarkers and therapeutic targets for IPF [10-12].

Animal models have been used in the preclinical setting to study the pathogenesis in IPF. We have developed a novel sheep model for lung fibrosis using bleomycin, which shares many of the characteristic features of IPF [13]. The model has been used to test and compare candidate anti-fibrotic drugs [14-16] to the FDA-approved drug pirfenidone (which is efficacious in the sheep model [17]. This is the first report evaluating the expression profiles of miRNAs under experimental conditions using the sheep model for lung fibrosis. After defining the miRNA expression profiles in the sheep model for lung fibrosis, these profiles were compared with those reported in the literature for IPF patients and in mouse lung fibrosis models. We also performed a pathway enrichment programs on the differentially expressed miRNA to illustrate the various biological mechanisms associated with lung fibrosis.

\section{Results}

\section{Segmental lung compliance reduced when the lung fibrosis increases at week 4}

Lung function was assessed by measuring the segmental compliance (Cseg) in saline- and bleomycin-infused lung lobes at baseline and week 4 (Fig. 1C). The mean segmental compliance was significantly reduced following the bleomycin infusion in lung segments at week 4 . In contrast, the lung compliance of the saline-infused control lung segments remained the same at the baseline and week 4 .

Our findings were further supported by the histopathology of lung segments stained with H \& E and Masson's trichrome stain. Moderate to severe irregular, multifocal collagen deposits were observed in the bleomycininfused sheep lung segments. These excessive collagen and connective tissue depositions resulted in irregular thickening of alveolar septa. In contrast, the saline infused lung segments displayed minimal fibrotic changes and were consistent with healthy lung tissues. The highest fibrosis score was observed in the lung segments infused with bleomycin when compared to saline lung segments (Fig. 1E and F). Furthermore, an assessment of Masson's trichrome-stained lung tissues showed that the percentage of blue (a measure of connective tissue content) was significantly increased in bleomycininfused lung segments compared to saline control segments (Fig. 1D and F).

\section{Differentially expressed miRNA profile in sheep model for lung fibrosis}

miRNA profiles were evaluated in 5 sheep, which were selected based on the low lung compliance and high fibrotic scores. The lung tissues were obtained to evaluate the changes in the miRNA expression levels at week 4 when the bleomycin injury is optimal. The data taken from 5 sheep showed that 49 miRNAs were differentially expressed due to bleomycin lung injury (Fig. 2). Out of those, 32 miRNAs were significantly downregulated in bleomycin-infused lung segments compared to the control/saline infused lung segments. In addition, $17 \mathrm{miR}$ NAs were significantly up regulated in bleomycininfused lung segments at week 4 when compared to saline infused lung segments (Fig. 2) (Table 1).

Four miRNAs - miR-29a, miR-26a, miR-30a and miR21 - that were differentially expressed in the miRNA microarray were examined by qPCR for validation. Bleomycin injury was associated with a significant downregulation of miR-30a, while miR-29a and miR-26a showed a similar but non-significant downregulation trend with bleomycin infusion (Fig. 3A, B \& C). Furthermore, bleomycin induced lung damage was associated with a significant upregulation of miR-21 expression (Fig. 3D) in sheep. Overall, for each of these miRNAs, the up- or downregulation expression pattern was similar in both the microarray and the qPCR assays.

Comparative evaluation of miRNA profiles showed that 6 miRNA families (miR-26, miR-29, miR-30, let-7, miR-17, miR-19) were downregulated in lung fibrosis for all three species; human, sheep, and mouse (Table 2 and Fig. 4A). In addition, 11 miRNA families were downregulated similarly in IPF patients and sheep models, but not in mice, while a different group of 11 miRNA families were downregulated in IPF patients and bleomycin damaged mouse lungs, but not in fibrotic sheep lungs (Table 2 and Fig. 4A).

Two miRNA families (miR-21 and miR-199) were upregulated in the two animal species (sheep and mouse), as well as in IPF patients, (Fig. 4B, Table 2). Furthermore, we identified 7 miRNA families where upregulation was restricted to IPF patients and sheep lung fibrosis (not mice), and a different set of 6 miRNA families where the upregulation was restricted to IPF patients and mice (not sheep) (Table 2 and Fig. 4B).

Overall, the sheep model and IPF patients had a total of 18 miRNAs in common that were differentially expressed, while the mouse model and IPF patients had a total of 17 miRNAs in common that were differentially expressed. Furthermore, around 98 miRNAs (Fig. 4) that 


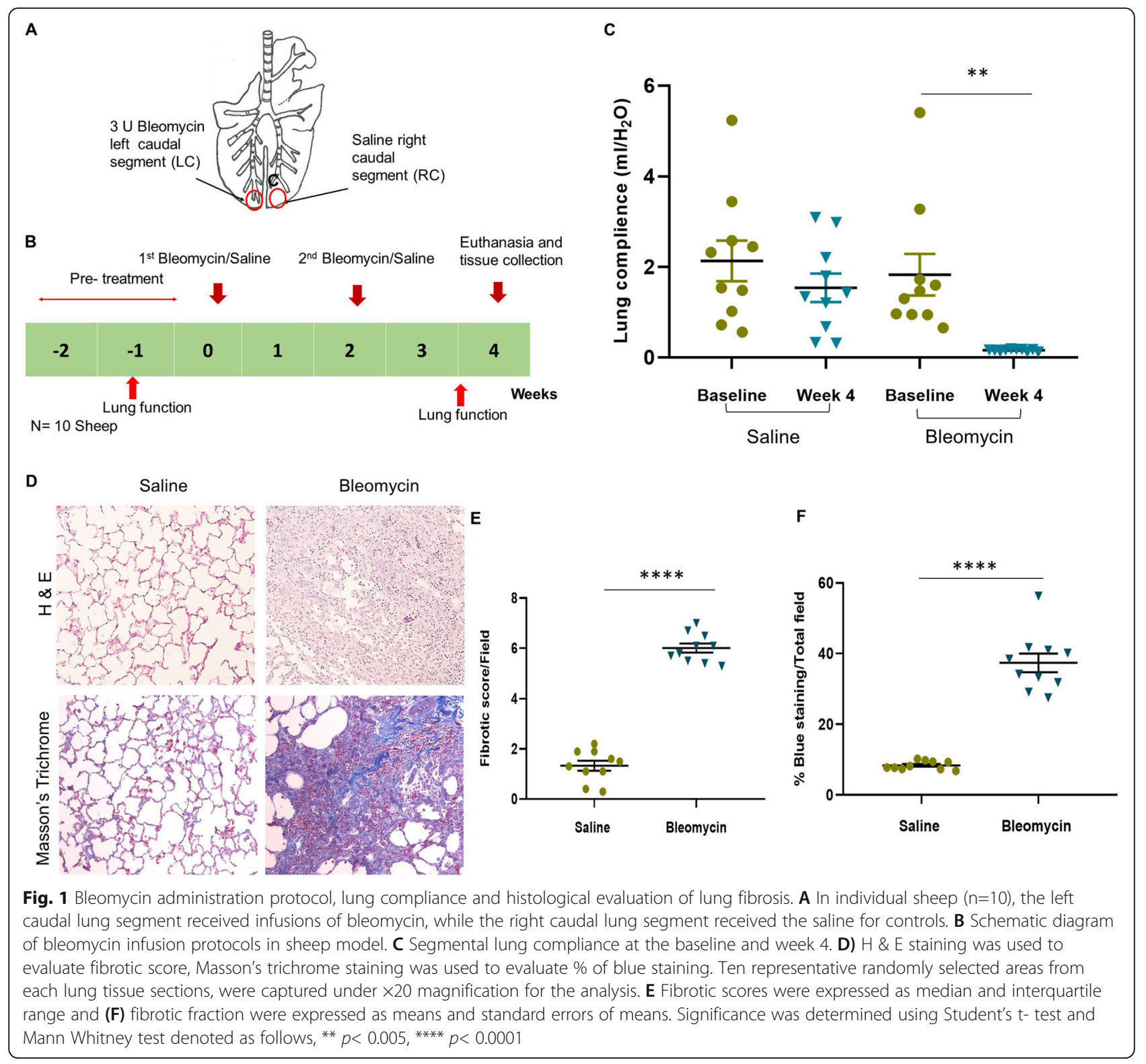

were detected in IPF patients were not detected in the sheep and mouse models of lung fibrosis.

\section{GO analysis and KEGG pathway analyses}

Pathway enrichment analyses were performed on 49 differentially expressed miRNAs in the sheep model for lung fibrosis using DIANA mirPath v.3 software. A total of 25 signaling pathways were identified as the most significantly enriched pathways according to their $\mathrm{p}$ - value $(p<0.05)$. According to the GO analysis, the differentially expressed miRNAs were mainly enriched for GO terms related to gene expression, extracellular matrix disassembly, neurotrophin TRK receptor signaling pathway, extracellular matrix organization, collagen catabolic process, endoplasmic reticulum lumen, extracellular matrix structural constituent and fibroblast growth factor receptor signaling pathways (Fig. 5).

KEGG pathway analysis was used to determine the biological pathways associated with the differentially expressed miRNAs in sheep model for lung fibrosis. KEGG pathway analysis demonstrated that the dysregulated miRNA could be enriched significantly into 29 signalling pathways according to their $p$-value $(p<0.05)$ (Fig. 6). PI3K-Akt signalling pathway, protein digestion and absorption, extracellular matrix receptor interaction, TGF- $\beta$ signalling pathway, miRNAs in cancer, small cell lung cancer, neurotrophin signalling pathway, p53 signalling pathway, platelet activation, apoptosis, and ErbB signalling pathway are some of the predominant pathways. We performed a comparative evaluation of 


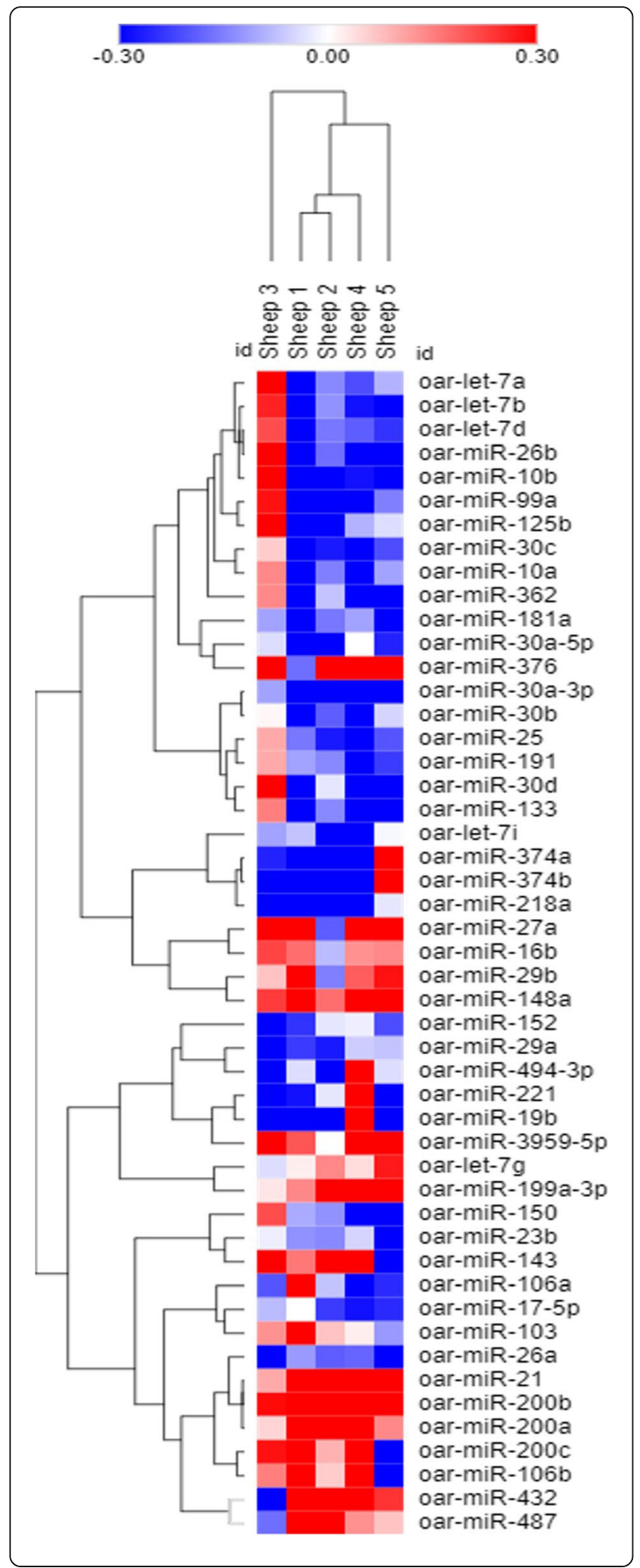

Fig. 2 Heatmap of significantly expressed miRNA microarray expression profile in the sheep model for lung fibrosis. The expression level of each miR was presented as fold-change relative to the control/saline group. Average intensity from the 21 replicates were taken for each miRNA probes tested for bleomycin and saline/ control lung segments. Student's t-test was performed and $p<0.01$ was considered statistically significant. Hierarchical clustering was performed by applying One minus Pearson correlation to cluster differentially expressed miRNAs. Blue indicates under expression and red indicates over expression

biological pathways enriched by differential expression of microRNAs in IPF patients and sheep and mice (Table 3).

\section{Discussion}

This study provides a comprehensive evaluation of the miRNA expression profile in a sheep model developed for lung fibrosis. We discovered a total of 49 differentially expressed miRNAs, 32 of which were significantly downregulated due to bleomycin induced lung injury, while 17 were significantly upregulated. Moreover, the differentially expressed miRNA families (miR-29, miR26, miR-30, let-7, miR-21, miR-19, miR-17, miR-199) reported for IPF patients, were also differentially expressed

Table 1 Significantly expressed microRNA microarray profile in sheep model for lung fibrosis

\begin{tabular}{ll}
\hline Downregulated miRNA & Upregulated miRNA \\
\hline oar-miR-181a & oar-miR-21 \\
oar-miR-23b & oar-miR-199a-3p \\
oar-let-7a & oar-miR-103 \\
oar-let-7b & oar-miR-29b \\
oar-let-7d & oar-let-7 g \\
oar-let-7i & oar-miR-27a \\
oar-miR-25 & oar-miR-200a \\
oar-miR-26a & oar-miR-200b \\
oar-miR-26b & oar-miR-200c \\
oar-miR-29a & oar-miR-432 \\
oar-miR-30a-5p & oar-miR-16b \\
oar-miR-30a-3p & oar-miR-106b \\
oar-miR-30b & oar-miR-148a \\
oar-miR-30c & oar-miR-376 \\
oar-miR-30d & oar-miR-143 \\
oar-miR-10a & oar-miR-487 \\
oar-miR-10b & oar-miR-3959-5p \\
oar-miR-99a & \\
oar-miR-150 & \\
oar-miR-152 & \\
oar-miR-374a & \\
oar-miR-374b & \\
oar-miR-133 & \\
oar-miR-218a & \\
oar-miR-125b & \\
oar-miR-191 & \\
oar-miR-106a & \\
oar-miR-221 & \\
oar-miR-362 & \\
oar-miR-494-3p & \\
oar-miR-17-5p & \\
oar-miR-19b & \\
\hline & \\
\hline
\end{tabular}


A

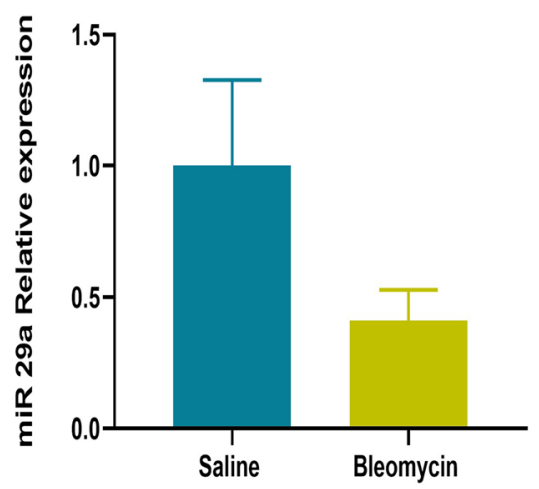

C

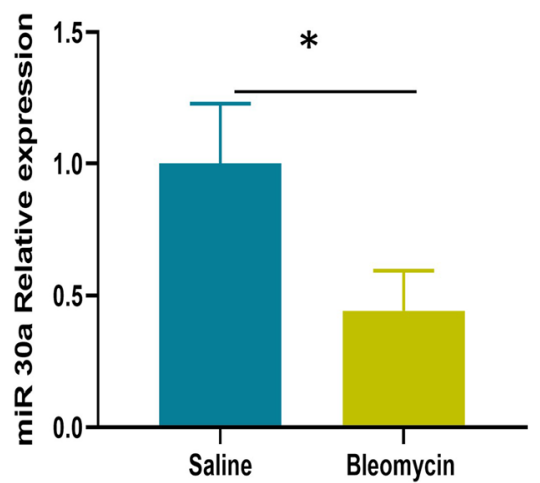

B

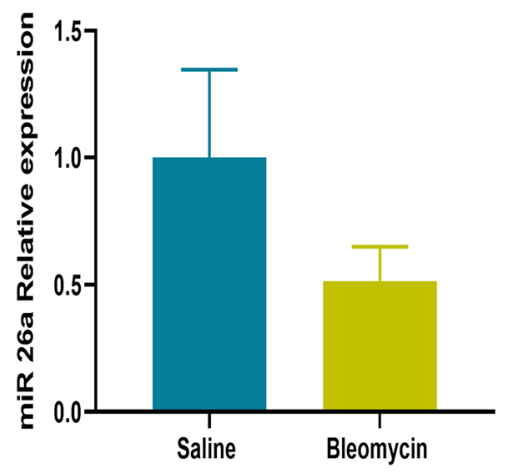

D

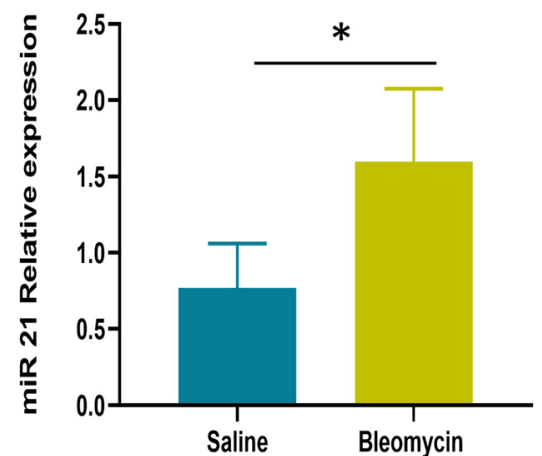

Fig. 3 Real-time PCR relative expression of miR-29a, miR-26a, miR-30a and miR-21 in sheep lung segments infused with bleomycin ( $n=5)$ and saline $(n=5)$. Significance was determined using student's t- test and denoted as follows, ${ }^{*} p<0.05$

in the bleomycin damaged lung tissues of both the sheep and mice $[4,19,32,38]$. In addition, we found $18 \mathrm{miR}$ NAs which were differentially expressed only in sheep and IPF fibrotic lungs, but not in bleomycin-injured mouse lungs. Pathway enrichment analyses of biological processes and signaling pathways of the 49 differentially expressed miRNAs, identified many pathways that were closely associated with IPF patients (Table 3) [11, 31, 36]. Moreover, these analyses revealed key miRNAs that play vital roles in important biological processes in fibrotic lungs. These processes include cell proliferation and apoptosis; cell movements; cellular metabolism; and intracellular signaling.

Amongst the 18 miRNAs that were differentially expressed in both the sheep model and IPF patients, but not in mice, 11 miRNAs; miR-181, miR-23, miR-25, miR-99, miR-150, miR-374, miR-125, miR-191, miR-221, miR-106 and miR-362 were similarly down-regulated and 7 miRNAs; miR-432, miR-487, miR-143, miR-376, miR-200b, miR-200c and miR-27 were up-regulated. Previous studies have investigated the mechanisms of some of these miRNAs. When miR-181 is transfection into Human Pulmonary Microvascular Endothelial cells (HPMEC) it was found to decrease Fas- induced apoptosis, while reducing inflammatory factors [39]. miR-23 has been found to be expressed in $\mathrm{T}$ cells and involved in the regulation of $\mathrm{T}$ cell function. Moreover, the aberrant expression of miR-23 resulted in the dysregulation of $\mathrm{T}$ cell activation and differentiation [40]. miR-374 can inhibit Fas-induced apoptosis in human primary retinal pigment epithelial (RPE) cells by targeting Fas during oxidative conditions. In particular, miR-374 overexpression restrained tumorigenicity and cell proliferation, while accelerating cell apoptosis through targeting Wnt16 and AKT1, which inhibits AKT signal pathway [41]. miRNA-221 targets several genes that are involved in the TGF- $\beta$ signaling, including JNK1 (c-Jun N-terminal kinase 1 ), TGF- $\beta$ receptor 1 and TGF- $\beta$ receptor 2 , and ETS-1 (ETS proto-oncogene 1) [42]. Inhibition of miR106 in Renal Cell Cancer cell lines altered the cell migration, invasion, and wound healing abilities [43]. miRNA miR-191 is expressed in $\mathrm{T}$ cells and acts as a key 
Table 2 Comparative analysis of microRNA in IPF patients and sheep and mouse models for lung fibrosis

\begin{tabular}{|c|c|c|c|c|}
\hline Dysfunction & $\begin{array}{l}\text { miRNAs in human, } \\
\text { sheep, and mice }\end{array}$ & $\begin{array}{l}\text { miRNAs in human } \\
\text { and sheep }\end{array}$ & $\begin{array}{l}\text { miRNAs in human } \\
\text { and mouse }\end{array}$ & Reference \\
\hline & let-7 & miR-181 & miR-27 & {$[1,2,6,8,12,18-33]$} \\
\hline & miR-29 & miR-23 & miR-130 & \\
\hline & miR-30 & $\operatorname{miR}-25$ & miR-326 & \\
\hline \multirow[t]{11}{*}{ Downregulated } & miR-26 & miR-99 & miR-18 & \\
\hline & miR-19 & miR-150 & miR-20 & \\
\hline & miR-17 & miR-374 & miR-92 & \\
\hline & & miR-125 & miR-200 & \\
\hline & & miR-191 & miR-157 & \\
\hline & & miR-106 & miR-140 & \\
\hline & & $\operatorname{miR}-221$ & miR-145 & \\
\hline & & miR-362 & miR-338 & \\
\hline & miR-21 & miR-432 & miR-142 & {$[1,2,6,8,18,19,23,25,26,28,31,33-35]$} \\
\hline & miR-199 & miR-376 & miR-155 & \\
\hline & & miR-487 & miR-449 & \\
\hline \multirow[t]{4}{*}{ Upregulated } & & miR-200b & miR-133 & \\
\hline & & miR-200c & miR-96 & \\
\hline & & miR-27 & miR-34 & \\
\hline & & miR-143 & & \\
\hline
\end{tabular}

A

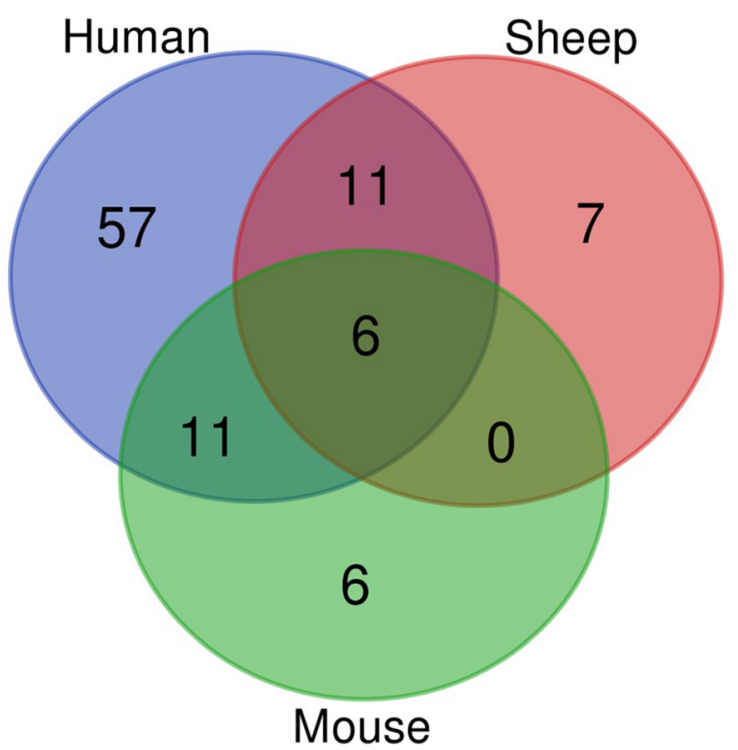

Fig. 4 Venn diagrams of differentially expressed microRNA in IPF patients and sheep and mouse models for lung fibrosis reported between the period of 2010-2020. Data were analysed using the Bioinformatic and Evolutionary Genomics software A) Down-regulated microRNA. B) Up-regulated microRNA

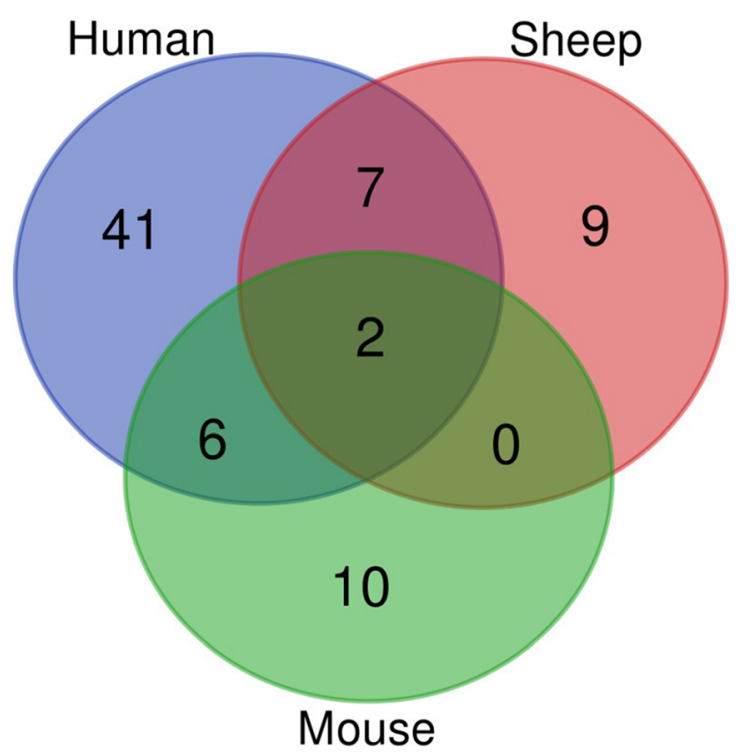

B 


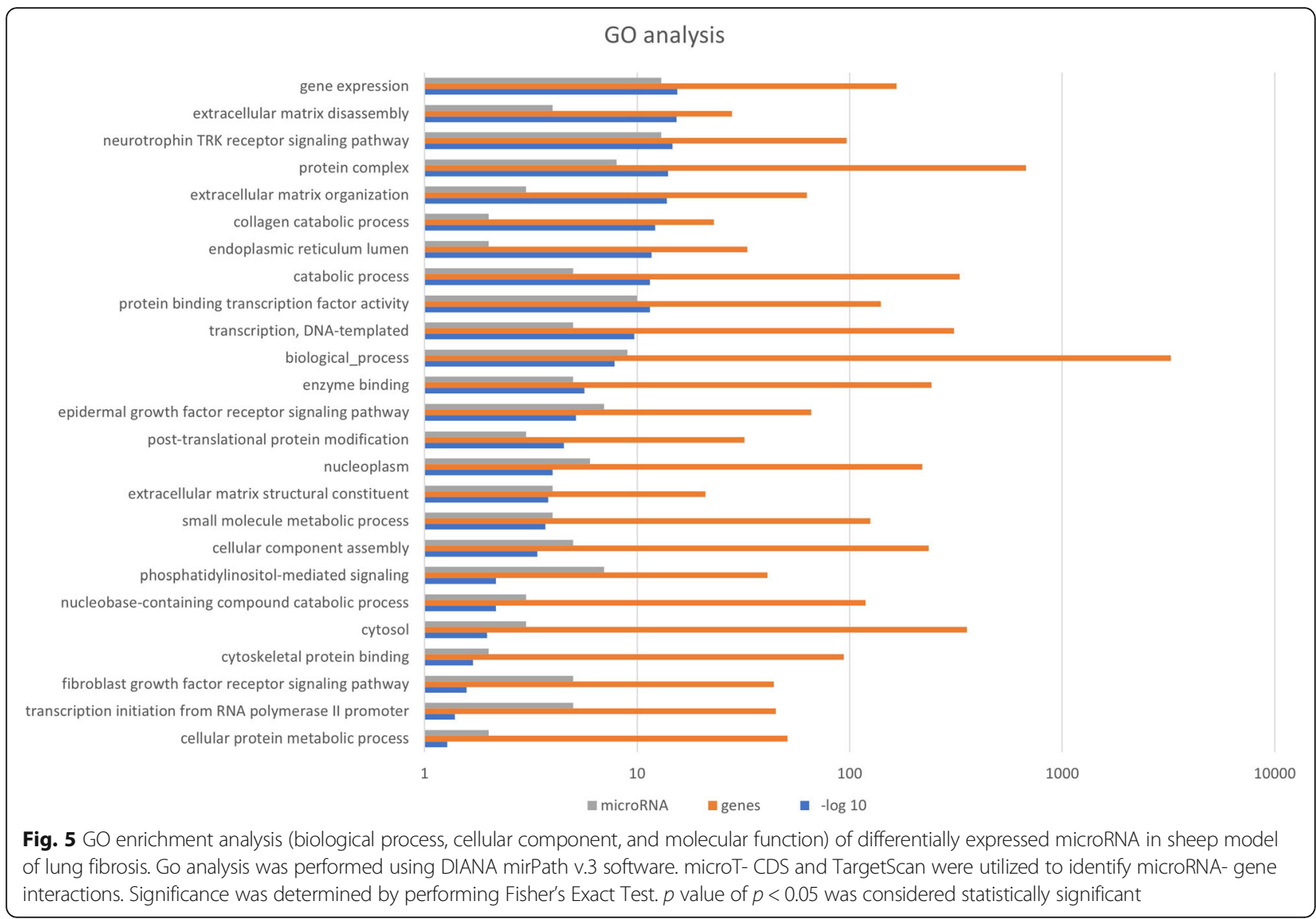

regulator of, memory, and regulatory $\mathrm{T}$ cell homeostasis [44]. While the above-mentioned studies give an insight into how these miRNAs might be operating, further investigations are needed to discover the contribution of these miRNAs to the pathogenesis of lung fibrosis, especially in larger animals.

While the alterations in the miRNA expression patterns have been discovered in IPF patients, the exact mechanisms of how these miRNA expressions change with the disease progression is still under investigation. Our findings suggest the molecular pathogenesis of the sheep bleomycin model for lung fibrosis shares some of the molecular mechanisms of IPF patients. This is an interesting finding due to the differences in age, duration of disease, etiology, and progressive nature of bleomycin induce lung fibrosis and IPF. Bleomycin induced lung fibrosis is an acute injury process with a known etiology, and apart from the recent exception [45], most animal models using bleomycin do not demonstrate the progressive nature of IPF [46]. In contrast, IPF is chronic and progressive disease condition with an unknown etiology. Despite these differences, both IPF and animal models of bleomycin-induced lung injury share common pathogenic mechanisms.
The miR-29, miR-26, miR-30, let-7, and miR-21 miRNA families were all differentially expressed in sheep, human and mice, and have been widely investigated as mediators in fibrotic disorders [5]. Previous studies showed that miR-29 is one of the main miRNAs involved in the pathogenesis of IPF [4, 47]. We showed that miR-29 is downregulated in the sheep model of lung fibrosis, similar to its expression patterns in IPF patients and fibrotic mouse lungs [48, 49]. Previous research showed that profibrotic factors such as transforming growth factor $\beta$ (TGF- $\beta$ ), platelet derived growth factor (PDGF), interleukin-4 (IL-4) and tumor necrosis factor $\alpha$ (TNF- $\alpha$ ) suppress miR-29 expression during the process of fibrosis [47]. Down regulation of miR-29 leads to overexpression of these profibrotic factors, causing further downregulation of miR-29 expression. This results in the promotion of collagen and extracellular matrix-related gene expression [5, 47]. Hence, miR-29 can directly regulate these vital processes that are important in the underlying mechanisms of pulmonary fibrosis [47].

miR-26a has been identified as potential therapeutic target that can be exploited to reduce fibrogenesis [18]. Expression of miR-26a was downregulated in bleomycin- 


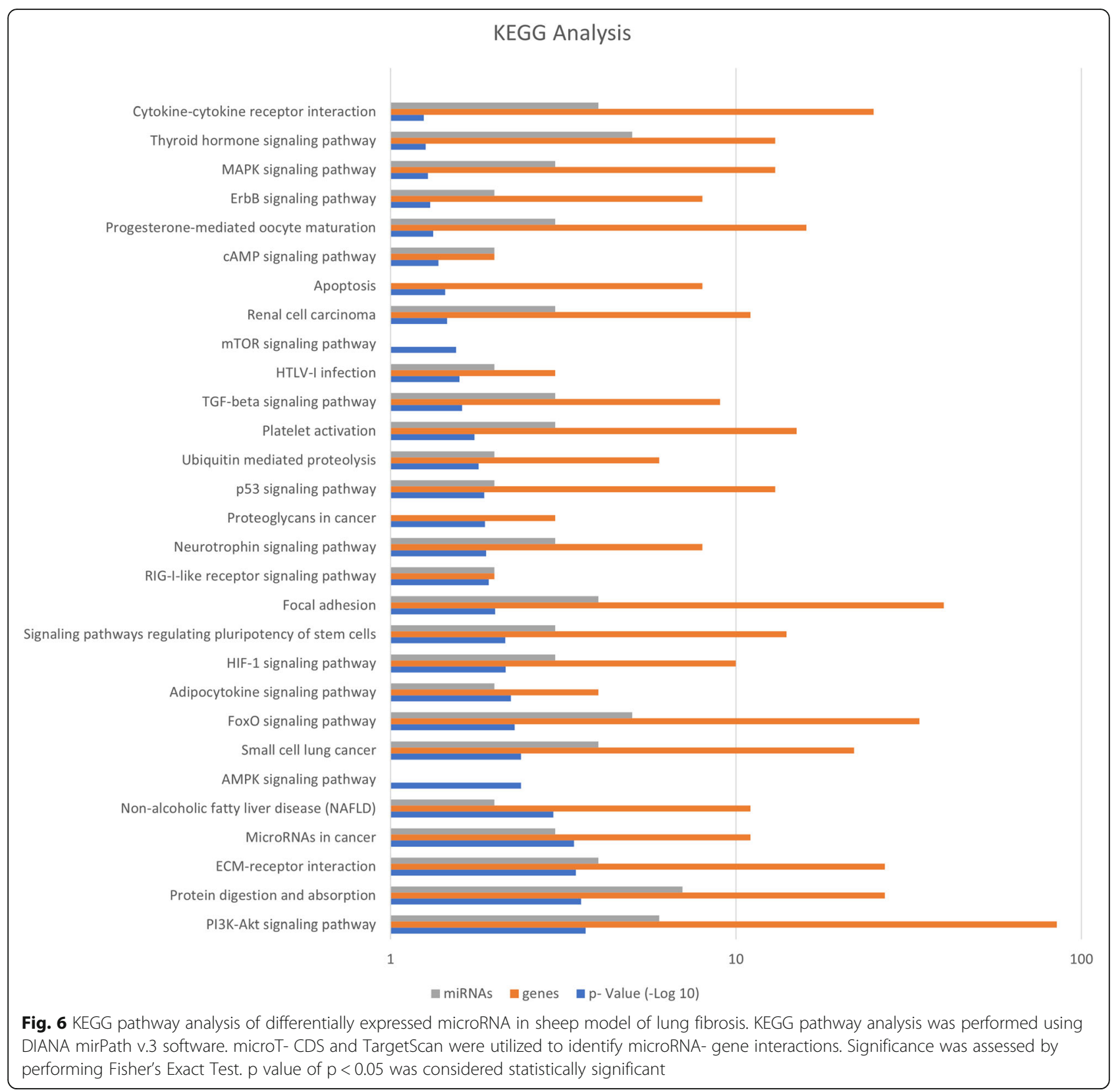

damaged sheep and mouse lungs, as it is in IPF patients. Downregulation of miR-26a is likely via TGF- $\beta 1$-induced activation of Smad3 [18]. Previous studies showed that miR-26a is involved in proliferation and differentiation of fibroblasts into myofibroblasts in $\operatorname{IPF}[12,18]$. Importantly, the overexpression of miR-26a attenuates bleomycin-induced pulmonary fibrosis in mice and TGF$\beta 1$-induced fibrogenesis in MRC-5 cells [18]. This suggests that it is a candidate for further investigation as a target to treat IPF [18].

Furthermore, we found that miR-30a expression was significantly downregulated due to bleomycin-induced lung injury in the sheep model. Our findings were in line with the low expression of miR-30a in IPF patients and mouse bleomycin models [38]. Previous studies showed that miR-30a was downregulated by $71.8 \%$ in the bronchoalveolar lavage fluids of IPF patients [38]. In the current study, KEGG analysis showed enrichment of TGF- $\beta$ and MAPK signaling pathways, which is consistent with the findings of Liu [38] in which they showed that the miR-30a may directly interact with TGF- $\beta$ activated kinase 1/MAP $3 \mathrm{~K} 7$ binding protein 3 (TAB3) mRNA. They also showed that overexpression of miR$30 \mathrm{a}$ attenuates TGF- $\beta 1$-induced upregulation of TAB3, $\alpha$-SMA and fibronectin expression in $293 \mathrm{~T}$ cells [38]. These studies suggest that miR-30a could be one of the 
Table 3 Comparative evaluation of biological pathways enriched by differential expression of microRNA

\begin{tabular}{|c|c|c|}
\hline Biological pathways enriched in IPF patients & $\begin{array}{l}\text { Sheep } \\
\text { model }\end{array}$ & $\begin{array}{l}\text { Mouse } \\
\text { model }\end{array}$ \\
\hline$[11,31,36]$ & & {$[26,37]$} \\
\hline TGF-beta signalling pathway & $\checkmark$ & $\checkmark$ \\
\hline MAPK signalling pathway & $\checkmark$ & $\checkmark$ \\
\hline PI3K-Akt signalling pathway & $\checkmark$ & \\
\hline \multicolumn{3}{|l|}{ Insulin signalling pathway } \\
\hline ErbB signalling pathway & $\checkmark$ & \\
\hline Focal adhesion & $\checkmark$ & $\checkmark$ \\
\hline Adherens junction & $\checkmark$ & $\checkmark$ \\
\hline Wnt signalling pathway & & $\checkmark$ \\
\hline \multicolumn{3}{|l|}{ RNA degradation } \\
\hline Ubiquitin mediated proteolysis & $\checkmark$ & \\
\hline \multicolumn{3}{|l|}{ Endocytosis } \\
\hline HIF-1 signalling pathway & $\checkmark$ & \\
\hline Neurotrophin signalling pathway & $\checkmark$ & $\checkmark$ \\
\hline Adipocytokine signalling pathway & $\checkmark$ & \\
\hline \multicolumn{3}{|l|}{ Regulation of actin cytoskeleton } \\
\hline \multicolumn{3}{|l|}{ Osteoclast differentiation } \\
\hline Hepatitis B & & $\checkmark$ \\
\hline Jak-STAT signalling pathway & & $\checkmark$ \\
\hline mTOR signalling pathway & $\checkmark$ & $\checkmark$ \\
\hline Notch signalling pathway & $\checkmark$ & \\
\hline HTLV-I infection & $\checkmark$ & \\
\hline Hedgehog signalling pathway & & $\checkmark$ \\
\hline \multicolumn{3}{|l|}{ Amoebiasis } \\
\hline \multicolumn{3}{|l|}{ T cell receptor signalling pathway } \\
\hline MicroRNAs in cancer & $\checkmark$ & $\checkmark$ \\
\hline Cell cycle & & $\checkmark$ \\
\hline \multicolumn{3}{|l|}{ Proteoglycans in cancer } \\
\hline Pathways in cancer & & $\checkmark$ \\
\hline Protein processing in endoplasmic reticulum & $\checkmark$ & \\
\hline Renal cell carcinoma & $\checkmark$ & $\checkmark$ \\
\hline \multicolumn{3}{|l|}{ Fatty acid metabolism } \\
\hline Hippo signalling pathway & & $\checkmark$ \\
\hline Nuerotrophin TRK receptor signalling pathway & $\checkmark$ & \\
\hline $\begin{array}{l}\text { Epidermal growth factor receptor signalling } \\
\text { pathway }\end{array}$ & $\checkmark$ & \\
\hline $\begin{array}{l}\text { Fibroblast growth factor receptor signalling } \\
\text { pathway }\end{array}$ & $\checkmark$ & \\
\hline ECM-receptor interaction & $\checkmark$ & \\
\hline p53 signalling pathway & $\checkmark$ & \\
\hline Gene expression & $\checkmark$ & \\
\hline Apoptosis & $\checkmark$ & $\checkmark$ \\
\hline \multicolumn{3}{|l|}{ Vascular smooth muscle contraction } \\
\hline \multicolumn{3}{|l|}{ GnRH signalling pathway } \\
\hline Pathways in cancer & $\checkmark$ & $\checkmark$ \\
\hline VEGF signalling pathway & $\checkmark$ & \\
\hline Calcium signalling pathway & & \\
\hline
\end{tabular}

key factors that supports progression of lung fibrosis in IPF patients.

Our study showed that miR-21 and miR-199 expression were upregulated in bleomycin-injured sheep and mouse lungs, consistent with findings in IPF lungs. High expression of miR-199 was observed in fibrotic foci of IPF lungs [50]. Furthermore, silencing miR-199a-5p strongly inhibited TGF- $\beta 1$-mediated differentiation of fibroblasts into myofibroblasts, wound repair and SMAD signaling in MRC-5 cells [50]. The importance of upregulated miRNAs in the fibrotic processes has been shown in studies where downregulation of miR-21 results in the attenuation of bleomycin-induced lung fibrosis in mice [35]. miR-21 is induced by the TGF- $\beta$ signaling pathway and amplifies TGF- $\beta$ signaling in a positive feedback fashion [35]. In addition, miR-21 promotes epithelial mesenchymal transition and enhances miR21 expression in myofibroblasts [34, 35]. For these reasons, miR-21 is considered as a potential therapeutic target for IPF [35].

Bleomycin-infused sheep lung segments also showed downregulation in the members of let-7 family, consistent with findings in IPF patients and mouse bleomycin models [27, 32, 51]. Let-7 expression is mainly localized to the alveolar epithelium in normal lungs and a significant reduction was observed in alveolar epithelium due to inhibition by the key profibrotic cytokine TGF- $\beta$ in IPF lungs [27]. Therefore, it has been identified as one of the key regulatory events responsible for the phenotypic changes occur in the alveolar epithelium of IPF lungs [27].

While this study used a microarray which profiles predefined transcripts to identify miRNAs involved in sheep lung fibrosis, future studies using whole transcriptome sequencing methods which capture the complete miRNA profile will enable the discovery of novel ovine miRNAs regulating fibrosis. Furthermore, mechanistic studies to delineate how the identified miRNAs regulate fibrotic genes are required.

Discovery of dysregulated miRNA expression profiles and the associated pathogenic mechanisms involved in the disease process of IPF potentially open new therapeutic avenues for IPF. In addition, miRNAs have also been considered as top potential biomarkers due to their upstream positions in regulation cascades which provide the benefit of an early diagnosis. While the alterations can be readily discovered by genomic tools such as microarrays and next generation sequencing, and even low level expression of miRNAs can be detected via qPCR which is much more accessible in a diagnostic setting [52].

\section{Conclusion}

The novel insights of this study showed similarities in the miRNA expression profiles in a sheep model for lung 
fibrosis and IPF patients, while illustrating the useful pathogenic mechanisms associated with lung fibrosis, suggest that bleomycin induced lung injuries in the sheep model share some of the molecular mechanisms associated with human IPF. Importantly, some miRNAs were significantly expressed only in the sheep model and IPF patients. Hence, the sheep model can be potentially used as an additional tool to characterize the underlying molecular mechanisms associated with lung fibrosis.

\section{Materials and methods Experimental design}

Healthy female merino sheep $(n=10) 9-12$ months old were used as subjects for the pulmonary fibrosis model. The experimental procedures and tissue sample collections were approved by Animal Experimentation Ethics Committee, University of Melbourne (Parkville, VIC, Australia), which adheres to the ARRIVE guidelines (https://arriveguidelines.org) and the Australian Code of Practice for the care and use of laboratory animals for scientific purposes.

\section{Bleomycin administration protocol}

Bleomycin was used to induce pulmonary fibrosis in the sheep. $5 \mathrm{ml}$ of $3 \mathrm{U}$ bleomycin/segment $(0.6 \mathrm{U} / \mathrm{ml}$ bleomycin) and $5 \mathrm{ml}$ of $0.9 \%$ sterile saline (control) were bronchoscopically administered to the left and the right caudal lung segments respectively, as described in our previous study [13] (Fig. 1A). The saline-infused lung segment serves as the internal control in the sheep model. Intra-bronchial bleomycin/saline were administered in two doses delivered 2 weeks apart to the appropriate lung segment via the biopsy port of the fiber-optic bronchoscope (Fig. 1B).

\section{Segmental lung function analysis}

Wedged-bronchoscope technique was used to measure the changes in the segmental lung compliance which occur due to bleomycin at baseline and week 4 as described previously [13] (Fig. 1C).

\section{Necropsy and tissue sampling}

Animals were euthanized at the end of week 4 by administering barbiturate (Lethabarb). Targeted lung segments were isolated during the necropsy and cannulated by exposing the bronchiole to inflate the segments. Next, a mixture of 1:1 optimal cutting temperature (OCT) compound and sterile PBS solution was injected to inflate the lung segment to approximately $20 \mathrm{~cm} / \mathrm{H}_{2} \mathrm{O}$ of pressure to preserve the tissue architecture during processing. Several serial transverse sections were collected from the inflated segments (less than $0.5 \mathrm{~cm}$ thick). The sections were then fixed in $10 \%$ neutral buffered formalin and processed in paraffin for histopathological analysis.

Lung samples that were collected for miRNA analysis were flash frozen in liquid Nitrogen and transferred to $-80^{\circ} \mathrm{C}$.

\section{Histopathological examination}

Paraffin-embedded tissue sections $(5 \mu \mathrm{m})$ were stained with Hematoxylin and Eosin Y (H \& E) for general histology and assessment of pathological changes (Fig. 1D). Collagen present in the lung parenchyma was assessed by paraffin sections stained with Masson's trichrome stain (Trichome stain kit, Abcam (ab150686)) according to manufacturer's instructions (Fig. 1D).

\section{Scoring fibrosis}

Fibrosis was assessed in the lung tissue sections of sheep according to the scale proposed by Hubner [53]. H \& Estained lung tissue sections were evaluated by capturing, 10 random representative, non-overlapping fields under $\times 20$ magnification. All the images taken from samples for scoring were double-blinded and performed by an experienced veterinary pathologist AS (Fig. 1E).

\section{Fibrosis fraction}

Collagen present in the lung parenchyma was evaluated from the lung tissue sections stained with Masson's trichrome stain. 10 representative randomly selected, nonoverlapping fields without large airways and blood vessels were captured under $\times 20$ magnification. The images were then analyzed using the computer software Image Pro ${ }^{\circ}$ Plus (Version 6.3.0.512 for Windows, Media Cybernetics, Rockville, Maryland, USA). Color selector tool was used to measure the area of tissue stained with blue (collagen) within the field. The fraction was obtained by dividing the blue color-stained tissue area by the total area of the field and expressed as means and standard errors of mean (Mean \pm SEM) (Fig. 1F).

\section{Statistical analysis}

Statistical analysis was performed using GraphPad Prism software, version 8.0.1 for Windows (GraphPad Software, La Jolla California, USA). Lung compliance and the fibrotic fraction were evaluated using Student's ttest. The degree of fibrosis was analysed using the Mann-Whitney test. The data were expressed as the mean \pm standard error of the mean (Mean \pm SEM). A $p$ value of less than $0.05(p<0.05)$ was considered as statistically significant.

\section{RNA extraction from sheep lung tissues}

Five sheep $(n=5)$ with the most severe lung fibrosis were selected based on lung function and lung pathology to evaluate the microRNA expression profiles. Total 
RNA extraction was performed using the miRNeasy Mini Kit according to manufactures instruction (QIAG EN Pty Ltd., Doncaster, Australia). RNA quality was determined by an Agilent Bioanalyzer 2100.

\section{microRNA microarray and data analysis}

A custom designed ovine miRNA microarray was performed by a service provider LC Sciences, Houston, USA. It contained 21 replicates of 152 unique probes and 55 control probes. The unique probes were derived from identified ovine miRNA sequences downloaded from miRBase (http://www.mirbase.org/) and the microarray included miRNA that have previously been shown to be involved in idiopathic pulmonary fibrosis. Data were background corrected and $\log 2$ transformed, with the microarray dataset entered into the GEO database at NCBI (GSE166682) https:/www.ncbi.nlm.nih.gov/geo/ query/acc.cgi?acc=GSE166682. Statistical analysis was performed for individual sheep separately due to the biological variation. Average intensities from the 21 replicates were taken for each miRNA probe tested in bleomycin and saline/control lung segments. Student's ttest was performed and $p<0.01$ was considered statistically significant. Log2 fold change of differentially expressed miRNA in each individual sheep was illustrated using a heatmap (Morpheus software; https:// software.broadinstitute.org/morpheus) (Fig. 2). Hierarchical clustering was then performed by applying One minus Pearson correlation to cluster differentially expressed miRNAs. Significant differentially expressed miRNA is shown in Table 1.

\section{Real-time PCR (qPCR)}

qPCR was performed on selected miRNA to validate the miRNA microarray. RNA was isolated using the miRNeasy Mini kit (QIAGEN Pty Ltd., Doncaster, Australia) as mentioned above. miRCURY LNA miRNA PCR starter Kit (QIAGEN Pty Ltd., Doncaster, Australia) was used to perform qPCR according to the manufacturer's instructions. Primer sequences of the customized miRCURY LNA miRNA PCR assays are listed in Table 4. qPCR parameters for 40 cycles were as follows: initial heat activation $95^{\circ} \mathrm{C}$ for $2 \mathrm{~min}$, denaturation $95^{\circ} \mathrm{C}$ for $10 \mathrm{~s}$ followed by combined annealing $56^{\circ} \mathrm{C}$ for $60 \mathrm{~s}$ (AriaMx Real- time PCR System). All reactions were

Table 4 Customized primer sequences miRCURY LNA miRNA PCR assay for sheep model of lung fibrosis

\begin{tabular}{ll}
\hline microRNA & Primer sequence \\
\hline miR-30a & 5'UGUAACAUCCUCGACUGGAAGC \\
miR-21 & 5'UAGCUUAUCAGACUGAUGUUGAC \\
miR-29a & 5'UAGCACCAUCUGAAUCGGUU \\
miR-26a & 5'UUCAAGUAAUCCAGGAUAGGCU \\
\hline
\end{tabular}

performed in a $10 \mu \mathrm{l}$ reaction volume. miR-103 was used as the housekeeping reference miRNA. Statistical analysis was performed using student's t-test and $p<0.05$ was considered statistically significant (Fig. 3).

\section{Comparative analysis of miRNA expression profiles of IPF patients, sheep, and mouse models}

Journal articles published in English during the period of January 2010 to June 2020 were used for the comparative analysis of the miRNA expression in human and mouse. A systematic search was performed using the search engines "Google Scholar", "PubMed" and University of Melbourne library catalogue, and the search strategy included the keywords of "microRNA", "Idiopathic pulmonary fibrosis", "human" and "mice". miRNA expression in IPF patients and in vivo expression in the mouse model were considered for this analysis. All the dysregulated miRNA reported in IPF patients, sheep and mouse models were analyzed using the Bioinformatic and Evolutionary Genomics software and presented as Venn diagrams (Fig. 4).

\section{Gene ontology and KEGG pathway analysis}

The biological function of differentially expressed miRNA was determined by Gene Ontology (GO) analysis. The GO terms consist of 3 parts: biological process, cellular component, and molecular function. GO analysis was performed using DIANA mirPath v.3 software [54] by considering the three components mentioned above. DIANA microT-CDS and TargetScan were used to identify miRNA-gene interactions, while the significance was assessed by performing Fisher's Exact Test. A $p$-value $<0.05$ was considered statistically significant (Fig. 5). Kyoto Encyclopedia of Genes and Genomes (KEGG) pathway analysis was also performed using DIANA mirPath v.3 software to evaluate the functions of differentially expressed miRNA and gene interactions. Significance was determined by performing Fisher's Exact Test. $p$ values $<0.05$ were considered statistically significant (Fig. 6).

\section{Abbreviations \\ IPF: Idiopathic pulmonary fibrosis; Cseg: Segmental compliance; miRNA: microRNA; GO: Gene Ontology; KEGG: Kyoto Encyclopedia of Genes and Genomes; HPMEC: Human Pulmonary Microvascular Endothelial cells; TGF- $\beta$ : Transforming growth factor $\beta_{\text {i; }}$ PDGF: Platelet derived growth factor; IL-4-: Interleukin-4; TNF-a: Tumor necrosis factor- $\alpha$}

\section{Acknowledgements}

Not applicable.

\section{Authors' contributions}

U.E.P was the principal researcher for the study. U.E.P and K.J.S successfully designed the study and U.E.P, H.B.D, K.J.S performed sheep experiments and evaluated lung function. U.E.P performed the laboratory work and statistical analyses. A. S assisted in histopathology. U.E.P and R. W performed the molecular analysis of this study. U.E.P and K.J.S prepared the first draft of the 
manuscript; and R. W, H.B.D, A. S and S.N.V.D critically revised the manuscript; All authors read and approved the final version of the manuscript.

\section{Funding}

Not applicable.

\section{Availability of data and materials}

The microarray data deposited in GEO database at NCBI (GSE166682) https:// www.ncbi.nlm.nih.gov/geo/query/acc.cgi?acc=GSE166682.

\section{Declarations}

\section{Ethics approval and consent to participate}

The experimental procedures and tissue sample collections were approved by Animal Experimentation Ethics Committee, University of Melbourne (Parkville, VIC, Australia), which adheres to the ARRIVE guidelines (https:// arriveguidelines.org) and the Australian Code of Practice for the care and use of laboratory animals for scientific purposes.

\section{Consent for publication}

\section{Not applicable.}

\section{Conflict of interest}

All authors declare that they have no conflicts of interest.

\section{Author details}

${ }^{1}$ School of Veterinary Science, The University of Melbourne, Parkville, VIC, Australia. ${ }^{2}$ Walter and Eliza Hall Institute of Medical Research, Parkville, Australia. ${ }^{3}$ School of Veterinary Science, The University of Melbourne, Werribee, VIC, Australia. ${ }^{4}$ Centre for Reproductive Health, Hudson Institute of Medical Research, Australia and Monash University, Clayton, VIC, Australia.

\section{Received: 25 April 2021 Accepted: 29 September 2021}

\section{Published online: 17 November 2021}

\section{References}

1. Oak SR, Murray L, Herath A, Sleeman M, Anderson I, Joshi AD, et al. A micro RNA processing defect in rapidly progressing idiopathic pulmonary fibrosis. PLoS One. 2011;6(6):e21253. https://doi.org/10.1371/journal.pone.0021253.

2. Miao C, Xiong Y, Zhang G, Chang J. MicroRNAs in idiopathic pulmonary fibrosis, new research progress and their pathophysiological implication. Exp Lung Res. 2018;44(3):178-90. https://doi.org/10.1080/01902148.2018.1455927.

3. Wang L, Huang W, Zhang L, Chen Q, Zhao H. Molecular pathogenesis involved in human idiopathic pulmonary fibrosis based on an integrated microRNAmRNA interaction network. Mol Med Rep. 2018;18(5):4365-73. https://doi.org/10.3892/mmr.2018.9456.

4. Pandit KV, Milosevic J, Kaminski N. MicroRNAs in idiopathic pulmonary fibrosis. Transl Res. 2011;157(4):191-9. https://doi.org/10.1016/j.trsl.2011.01.012.

5. Stolzenburg LR, Harris $A$. The role of microRNAs in chronic respiratory disease: recent insights. Biol Chem. 2018;399(3):219-34. https://doi.org/10.1 515/hsz-2017-0249

6. Mizuno K, Mataki H, Seki N, Kumamoto T, Kamikawaji K, Inoue H. MicroRNAs in non-small cell lung cancer and idiopathic pulmonary fibrosis. J Hum Genet. 2017;62(1):57-65. https://doi.org/10.1038/jhg.2016.98.

7. Rajasekaran S, Rajaguru P, Sudhakar Gandhi PS. MicroRNAs as potential targets for progressive pulmonary fibrosis. Front Pharmacol. 2015;6:254.

8. Huang C, Yang Y, Liu L. Interaction of long noncoding RNAs and microRNAs in the pathogenesis of idiopathic pulmonary fibrosis. Physiol Genomics. 2015:47(10):463-9. https://doi.org/10.1152/physiolgenomics.00064.2015.

9. Pandit KV, Milosevic J. MicroRNA regulatory networks in idiopathic pulmonary fibrosis. Biochem Cell Biol. 2015;93(2):129-37. https://doi.org/1 0.1139/bcb-2014-0101

10. Li $P$, et al. Expression analysis of serum microRNAs in idiopathic pulmonary fibrosis. Int J Mol Med. 2014;33(6):1554-62. https://doi.org/10.3892/ijmm.2 014.1712 .

11. Yang G, Yang L, Wang W, Wang J, Wang J, Xu Z. Discovery and validation of extracellular/circulating microRNAs during idiopathic pulmonary fibrosis disease progression. Gene. 2015;562(1):138-44. https://doi.org/10.1016/j. gene.2015.02.065.

12. Li H, Zhao $X$, Shan $H$, Liang H. MicroRNAs in idiopathic pulmonary fibrosis: involvement in pathogenesis and potential use in diagnosis and therapeutics. Acta Pharm Sin B. 2016;6(6):531-9. https://doi.org/10.1016/j.a psb.2016.06.010

13. Organ L, Bacci B, Koumoundouros E, Barcham G, Kimpton W, Nowell CJ, et al. A novel segmental challenge model for bleomycin-induced pulmonary fibrosis in sheep. Exp Lung Res. 2015;41(3):115-34. https://doi. org/10.3109/01902148.2014.985806.

14. Organ L, Bacci B, Koumoundouros E, Kimpton WG, Samuel CS, Nowell CJ, et al. Inhibition of the K (ca)3.1 channel alleviates established pulmonary fibrosis in a large animal model. Am J Respir Cell Mol Biol. 2017;56(4):53950. https://doi.org/10.1165/rcmb.2016-00920C.

15. Perera UE, et al. Increased Levels of ER Stress and Apoptosis in a Sheep Model for Pulmonary Fibrosis Are Alleviated by In Vivo Blockade of the KCa3.1 Ion Channel. Can Respir J. 2021:2021:6683195.

16. Derseh HB, et al. KCa3.1 channel blockade attenuates microvascular remodelling in a large animal model of bleomycin-induced pulmonary fibrosis. Sci Rep. 2019;9(1):19893.

17. Dewage SNV, Organ L, Koumoundouros E, Derseh HB, Perera KUE, Samuel CS, et al. The efficacy of pirfenidone in a sheep model of pulmonary fibrosis. Exp Lung Res. 2019:45(9-10):310-22. https://doi.org/10.1080/01 902148.2019.1695019.

18. Liang H, Xu C, Pan Z, Zhang Y, Xu Z, Chen Y, et al. The antifibrotic effects and mechanisms of microRNA-26a action in idiopathic pulmonary fibrosis. Mol Ther. 2014;22(6):1122-33. https://doi.org/10.1038/mt.2014.42.

19. Liang H, Gu Y, Li T, Zhang Y, Huangfu L, Hu M, et al. Integrated analyses identify the involvement of microRNA-26a in epithelial-mesenchymal transition during idiopathic pulmonary fibrosis. Cell Death Dis. 2014;5(5): e1238. https://doi.org/10.1038/cddis.2014.207.

20. Das S, Kumar M, Negi V, Pattnaik B, Prakash YS, Agrawal A, et al. MicroRNA326 regulates profibrotic functions of transforming growth factor-beta in pulmonary fibrosis. Am J Respir Cell Mol Biol. 2014;50(5):882-92. https://doi. org/10.1165/rcmb.2013-01950C.

21. Yang $S$, Cui $H$, Xie N, Icyuz M, Banerjee $S$, Antony VB, et al. miR-145 regulates myofibroblast differentiation and lung fibrosis. FASEB J. 2013;27(6): 2382-91. https://doi.org/10.1096/fj.12-219493.

22. Wang C, Song X, Li Y, Han F, Gao S, Wang X, et al. Low-dose paclitaxel ameliorates pulmonary fibrosis by suppressing TGF-beta1/Smad3 pathway via miR-140 upregulation. PLoS One. 2013;8(8):e70725. https://doi.org/10.13 71/journal.pone.0070725.

23. Lino Cardenas CL, Kaminski N, Kass DJ. Micromanaging microRNAs: using murine models to study microRNAs in lung fibrosis. Drug Discov Today Dis Models. 2013;10(3):e145-51. https://doi.org/10.1016/j.ddmod.2012.11.003.

24. Dakhlallah D, Batte $K$, Wang Y, Cantemir-Stone CZ, Yan P, Nuovo G, et al. Epigenetic regulation of miR-17 92 contributes to the pathogenesis of pulmonary fibrosis. Am J Respir Crit Care Med. 2013;187(4):397-405. https:// doi.org/10.1164/rccm.201205-08880C

25. Yang S, Banerjee S, de Freitas A, Sanders YY, Ding Q, Matalon S, et al. Participation of miR-200 in pulmonary fibrosis. Am J Pathol. 2012;180(2): 484-93. https://doi.org/10.1016/j.ajpath.2011.10.005.

26. Honeyman L, Bazett M, Tomko TG, Haston CK. MicroRNA profiling implicates the insulin-like growth factor pathway in bleomycin-induced pulmonary fibrosis in mice. Fibrogenesis Tissue Repair. 2013;6(1):16. https://doi.org/1 0.1186/1755-1536-6-16.

27. Pandit KV, Corcoran D, Yousef H, Yarlagadda M, Tzouvelekis A, Gibson KF, et al. Inhibition and role of let-7d in idiopathic pulmonary fibrosis. Am J Respir Crit Care Med. 2010;182(2):220-9. https://doi.org/10.1164/rccm.2 00911-16980C.

28. Nho RS. Alteration of aging-dependent MicroRNAs in idiopathic pulmonary fibrosis. Drug Dev Res. 2015;76(7):343-53. https://doi.org/10.1002/ddr.21272.

29. Cui H, Banerjee S, Xie N, Ge J, Liu RM, Matalon S, et al. MicroRNA-27a-3p is a negative regulator of lung fibrosis by targeting Myofibroblast differentiation. Am J Respir Cell Mol Biol. 2016;54(6):843-52. https://doi.org/10.1165/rcmb.2 015-02050C.

30. Zhang S, Liu H, Liu Y, Zhang J, Li H, Liu W, et al. miR-30a as Potential Therapeutics by Targeting TET1 through Regulation of Drp-1 Promoter Hydroxymethylation in Idiopathic Pulmonary Fibrosis. Int J Mol Sci. 2017; 18(3):633. https://doi.org/10.3390/ijms18030633.

31. Fan $L$, et al. Analysis of microarray-identified genes and MicroRNAs associated with idiopathic pulmonary fibrosis. Mediat Inflamm. 2017;2017: 1804240.

32. Elliot S, Periera-Simon S, Xia X, Catanuto P, Rubio G, Shahzeidi S, et al. MicroRNA let-7 downregulates ligand-independent estrogen receptor- 
mediated male-predominant pulmonary fibrosis. Am J Respir Crit Care Med. 2019;200(10):1246-57. https://doi.org/10.1164/rccm.201903-05080C

33. Rezaei S, Mahjoubin-Tehran M, Aghaee-Bakhtiari SH, Jalili A, Movahedpour A, Khan $\mathrm{H}$, et al. Autophagy-related MicroRNAs in chronic lung diseases and lung cancer. Crit Rev Oncol Hematol. 2020;153:103063. https://doi.org/10.1 016/.j.critrevonc.2020.103063.

34. Yamada $\mathrm{M}$, et al. The increase of microRNA-21 during lung fibrosis and its contribution to epithelial-mesenchymal transition in pulmonary epithelial cells. Respir Res. 2013;14(1):95. https://doi.org/10.1186/1465-9921-14-95.

35. Liu G, Friggeri A, Yang Y, Milosevic J, Ding Q, Thannickal VJ, et al. miR-21 mediates fibrogenic activation of pulmonary fibroblasts and lung fibrosis. J Exp Med. 2010;207(8):1589-97. https://doi.org/10.1084/jem.20100035.

36. Cho JH, Gelinas R, Wang K, Etheridge A, Piper MG, Batte K, et al. Systems biology of interstitial lung diseases: integration of mRNA and microRNA expression changes. BMC Med Genet. 2011;4(1):8. https://doi.org/10.1186/1 755-8794-4-8.

37. Liu X, Liu H, Jia X, He R, Zhang X, Zhang W. Changing Expression Profiles of Messenger RNA, MicroRNA, Long Non-coding RNA, and Circular RNA Reveal the Key Regulators and Interaction Networks of Competing Endogenous RNA in Pulmonary Fibrosis. Front Genet. 2020;11:558095. https://doi.org/1 0.3389/fgene.2020.558095.

38. Yang $\mathrm{H}$, Liu X, Hu G, Xie Y, Lin S, Zhao Z, et al. Identification and analysis of microRNAs-mRNAs pairs associated with nutritional status in seasonal sheep. Biochem Biophys Res Commun. 2018;499(2):321-7. https://doi.org/1 0.1016/.jbbrc.2018.03.155.

39. Liu Y, Wang X, Li P, Zhao Y, Yang L, Yu W, et al. Targeting MALAT1 and miRNA-181a-5p for the intervention of acute lung injury/acute respiratory distress syndrome. Respir Res. 2021;22(1):1. https://doi.org/10.1186/s12931020-01578-8.

40. Cho S, Wu CJ, Yasuda T, Cruz LO, Khan AA, Lin LL, et al. miR-23 approximately 27 approximately 24 clusters control effector $T$ cell differentiation and function. J Exp Med. 2016;213(2):235-49. https://doi.org/1 0.1084/jem.20150990.

41. Tasharrofi N, Kouhkan F, Soleimani M, Soheili ZS, Kabiri M, Mahmoudi Saber $M$, et al. Survival improvement in human retinal pigment epithelial cells via Fas receptor targeting by miR-374a. J Cell Biochem. 2017;118(12):4854-61. https://doi.org/10.1002/jcb.26160.

42. Verjans $R$, Peters $T$, Beaumont FJ, van Leeuwen $R$, van Herwaarden $T$, Verhesen W, et al. MicroRNA-221/222 family counteracts myocardial fibrosis in pressure overload-induced heart failure. Hypertension. 2018;71(2):280-8. https://doi.org/10.1161/HYPERTENSIONAHA.117.10094.

43. Pan YJ, Wei LL, Wu XJ, Huo FC, Mou J, Pei DS. MiR-106a-5p inhibits the cell migration and invasion of renal cell carcinoma through targeting PAK5. Cell Death Dis. 2017;8(10):e3155. https://doi.org/10.1038/cddis.2017.561.

44. Lykken EA, Li QJ. The MicroRNA miR-191 supports T cell survival following common gamma chain signaling. J Biol Chem. 2016;291(45):23532-44. https://doi.org/10.1074/jbc.M116.741264.

45. Redente EF, Black BP, Backos DS, Bahadur AN, Humphries SM, Lynch DA, et al. Persistent, progressive pulmonary fibrosis and epithelial remodeling in mice. Am J Respir Cell Mol Biol. 2021;64(6):669-76. https://doi.org/10.1165/ rcmb.2020-0542MA.

46. Liu T, De Los Santos FG, Phan SH. The bleomycin model of pulmonary fibrosis. Methods Mol Biol. 2017;1627:27-42. https://doi.org/10.1007/978-1-4 939-7113-8_2

47. Cushing $L$, Kuang P, Lu J. The role of miR-29 in pulmonary fibrosis. Biochem Cell Biol. 2015;93(2):109-18. https://doi.org/10.1139/bcb-2014-0095.

48. Bibaki E, Tsitoura E, Vasarmidi E, Margaritopoulos G, Trachalaki A, Koutoulaki C, et al. miR-185 and miR-29a are similarly expressed in the bronchoalveolar lavage cells in IPF and lung cancer but common targets DNMT1 and COL1A1 show disease specific patterns. Mol Med Rep. 2018;17(5):7105-12. https://doi.org/10.3892/mmr.2018.8778.

49. Tang Y, He R, An J, Deng P, Huang L, Yang W. The effect of H19-miR-29b interaction on bleomycin-induced mouse model of idiopathic pulmonary fibrosis. Biochem Biophys Res Commun. 2016;479(3):417-23. https://doi. org/10.1016/j.bbrc.2016.09.028.

50. Lino Cardenas CL, et al. miR-199a-5p Is upregulated during fibrogenic response to tissue injury and mediates TGFbeta-induced lung fibroblast activation by targeting caveolin-1. PLoS Genet. 2013;9(2):e1003291.

51. Huleihel L, Ben-Yehudah A, Milosevic J, Yu G, Pandit K, Sakamoto K, et al. Let-7d microRNA affects mesenchymal phenotypic properties of lung fibroblasts. Am J Physiol Lung Cell Mol Physiol. 2014;306(6):L534-42. https:// doi.org/10.1152/ajplung.00149.2013.

52. Li Y, Kowdley KV. MicroRNAs in common human diseases. Genomics Proteomics Bioinformatics. 2012;10(5):246-53. https://doi.org/10.1016/j.gpb.2 012.07.005.

53. Hubner RH, et al. Standardized quantification of pulmonary fibrosis in histological samples. Biotechniques. 2008;44(4):507-11, 514-7. https://doi. org/10.2144/000112729.

54. Vlachos IS, Zagganas K, Paraskevopoulou MD, Georgakilas G, Karagkouni D, Vergoulis T, et al. DIANA-miRPath v3.0: deciphering microRNA function with experimental support. Nucleic Acids Res. 2015:43(W1):W460-6. https://doi. org/10.1093/nar/gkv403.

\section{Publisher's Note}

Springer Nature remains neutral with regard to jurisdictional claims in published maps and institutional affiliations.
Ready to submit your research? Choose BMC and benefit from:

- fast, convenient online submission

- thorough peer review by experienced researchers in your field

- rapid publication on acceptance

- support for research data, including large and complex data types

- gold Open Access which fosters wider collaboration and increased citations

- maximum visibility for your research: over $100 \mathrm{M}$ website views per year

At BMC, research is always in progress.

Learn more biomedcentral.com/submissions 